\section{Strengths and limitations of estimated and measured GFR}

\author{
Andrew S. Levey $(\mathbb{D}$, Josef Coresh, Hocine Tighiouart, Tom Greene and \\ Lesley A. Inker
}

Evaluation of glomerular filtration rate (GFR) is central to medical practice, research and public health. As investigators who developed the Modification of Diet in Renal Disease (MDRD) and Chronic Kidney Disease Epidemiology Collaboration (CKD-EPI) equations for estimation of GFR, and as leaders of national and international guideline committees and workshops on the use of estimated GFR (eGFR), we take issue with the conclusions of Porrini et al. stated in their Perspectives article (Porrini, E. et al. Estimated GFR: time for a critical appraisal. Nat. Rev. Nephrol. 15, 177-190 (2019) $\left.{ }^{1}\right)$, which discusses the reliability of eGFR equations. These authors conclude that "eGFR is an unreliable tool to assess renal function in health and disease, as well as in clinical practice and research". Instead of eGFR, they recommend more frequent use of measured GFR (mGFR). We agree that eGFR may be imprecise; using the most accurate eGFR equations, the proportion of eGFR values that are within 30\% of mGFR values $\left(\mathrm{P}_{30}\right)$ generally does not exceed $90 \%$, which is the performance goal for $\mathrm{eGFR}^{2,3}$. We also agree that more frequent use of mGFR is desirable, and we applaud the work of Porrini et al. in implementing plasma clearance of iohexol in research studies. However, we regard their conclusions as flawed for several reasons.

First, their analysis includes studies that did not standardize assays for creatinine or cystatin $\mathrm{C}$, leading to unpredictable bias in eGFR values based on levels of creatinine $\left(\mathrm{eGFR}_{\mathrm{cr}}\right)$, cystatin $\mathrm{C}\left(\mathrm{eGFR}_{\text {cys }}\right)$ or both $\left(\mathrm{eGFR}_{\text {cr-cys }}\right)$, and to unreliable estimates of the accuracy of these equations. This flaw can only be avoided by limiting the analysis to studies that used standardized assays and equations developed or re-expressed for use with standardized assays ${ }^{4}$.

Second, they underestimate error in mGFR compared with 'true' GFR', which contributes substantially to the errors reported in comparisons between eGFR and mGFR in both cross-sectional and longitudinal studies. A systematic review by Soveri and colleagues ${ }^{6}$ demonstrated bias and imprecision in all current mGFR methods compared to the classical method of inulin clearance described by Homer Smith; $\mathrm{P}_{30}$ often did not exceed $90 \%$. Even the classical inulin clearance method is imprecise, with a coefficient of variation $(\mathrm{CV})$ for repeated measures of approximately $7 \%$. The smallest reported CVs using other mGFR methods are approximately 5-15\%, generally with higher values for urinary clearance than plasma clearance methods ${ }^{8-14}$. Critically, for an unbiased mGFR method, a CV for repeated measures of $10 \%$ would be equivalent to approximately $90 \%$ of mGFR within $15 \%$ of true GFR $\left(\mathrm{P}_{15}\right.$ of $\left.90 \%\right)$.

Third, they propose an alternative performance goal for eGFR of $\mathrm{P}_{10}>90 \%$ compared with mGFR (90\% of eGFR values within $10 \%$ of mGFR values). This goal is unrealistic, as it is not attainable for repeated measurements of mGFR using many methods.

Fourth, they fail to consider that clinical decision-making in most settings includes multiple factors in addition to eGFR ${ }^{15,16}$. For many clinical decisions, a $\mathrm{P}_{30}$ for eGFR compared with mGFR of $80-90 \%$ is adequate. If more accurate assessment is required, $\mathrm{eGFR}_{\text {cys }}, \mathrm{eGFR}_{\text {cr-cys }}$ or $\mathrm{mGFR}$ are recommended as confirmatory tests, depending on their availability and the level of accuracy required for clinical decision-making.

In contrast to Porrini and colleagues, we conclude that both mGFR and eGFR have strengths and limitations and both can be improved. We recommend that clinicians, researchers and public health officials understand these strengths and limitations for wise use of both mGFR and eGFR.

There is a reply to this letter by Porrini, E. et al. Nat. Rev. Nephrol. https://doi.org/ 10.1038/s41581-019-0214-8 (2019).

Andrew S. Levey iD $^{1 *}$, Josef Coresh ${ }^{2}$,
Hocine Tighiouart ${ }^{3}$, Tom Greene ${ }^{4}$ and Lesley A. Inker

Division of Nephrology, Tufts Medical Center, Boston, MA, USA

${ }^{2}$ Department of Epidemiology, Johns Hopkins Bloomberg School of Public Health, Baltimore, MD, USA

${ }^{3}$ Institute for Clinical Research and Health Policy Studies, Tufts Medical Center, Boston, MA, USA
${ }^{4}$ Division of Epidemiology, Department of Internal Medicine, University of Utah, Salt Lake City, UT, USA. *e-mail: alevey@tuftsmedicalcenter.org https://doi.org/10.1038/s41581-019-0213-9

1. Porrini, E. et al. Estimated GFR: time for a critical appraisal. Nat. Rev. Nephrol. 15, 177-190 (2019).

2. National Kidney Foundation. K/DOOI clinical practice guidelines for chronic kidney disease: evaluation, classification, and stratification. Am. J. Kidney Dis. 39 (2 Suppl 1), 1-266 (2002)

3. Kidney Disease: Improving Global Outcomes (KDIGO) KDIGO 2012 Clinical Practice Guideline for the Evaluation and Management of Chronic Kidney Disease. Kidney Int. Suppl. 3, 1-150 (2013).

4. Myers, G. L. et al. Recommendations for improving serum creatinine measurement: a report from the Laboratory Working Croup of the National Kidney Disease Education Program. Clin. Chem. 52, 5-18 (2006).

5. Levey, A. S. \& Inker, L. A. GFR as the "gold standard": estimated, measured, and true. Am. J. Kidney Dis. 67 9-12 (2016).

6. Soveri, I. et al. Measuring GFR: a systematic review. Am. J. Kidney Dis. 64, 411-424 (2014).

7. Davies, D. F. \& Shock, N. W. The variability of measurement of inulin and diodrast tests of kidney function. J. Clin Invest 29, 491-495 (1950).

8. Levey, A. S. et al. Glomerular filtration rate measurements in clinical trials. Modification of Diet in Renal Disease Study Group and the Diabetes Control and Complications Trial Research Group. J. Am. Soc Nephrol 4 1159-1171 (1993).

9. Florijn, K. W. et al. Glomerular filtration rate measurement by "single-shot" injection of inulin Kidney Int. 46, 252-259 (1994).

10. Chowdhury, T. A. et al. Glomerular filtration rate determination in diabetic patients using iohexol clearance - comparison of single and multiple plasma sampling methods. Clin. Chim. Acta 277, 153-158 (1998).

11. Gaspari, F. et al. Precision of plasma clearance of iohexol for estimation of GFR in patients with renal disease. J. Am. Soc. Nephrol 9, 310-313 (1998).

12. Tan, G. D. et al. Clinical usefulness of cystatin C for the estimation of glomerular filtration rate in type 1 diabetes: reproducibility and accuracy compared with standard measures and iohexol clearance. Diabetes Care 25, 2004-2009 (2002).

13. Agarwal, R. Ambulatory GFR measurement with cold iothalamate in adults with chronic kidney disease. Am. J. Kidney Dis. 41, 752-759 (2003).

14. Rowe, C et al. Biological variation of measured and estimated glomerular filtration rate in patients with chronic kidney disease. Kidney Int. 96, 429-435 (2019).

15. Huang, N. et al. Estimated GFR for living kidney donor evaluation. Am. J. Transplant. 16, 171-180 (2016)

16. Grams, M. E. et al. Predicting timing of clinical outcomes in patients with chronic kidney disease and severely decreased glomerular filtration rate. Kidney Int. 93, 1442-1451 (2018).

\section{Acknowledgements}

We acknowledge the assistance of J. Chaudhari (Tufts Medical Center, Boston, MA, USA) in manuscript preparation.

\section{Competing interest}

A.S.L. has received grants from the US National Institutes of Health (NIH) and the National Kidney Foundation (NKF) for research related to eGFR equations. J.C. has received grants from the $\mathrm{NIH}$ and the NKF, some of which are related to the development of eGFR equations. T.G. reports grants from the NKF and personal fees from DURECT Corporation, Janssen Pharmaceuticals and Pfizer Inc. L.A.I. reports funding awarded to Tufts Medical Center for research and contracts with the NIH, NKF, Retrophin, Omeros, Reata Pharmaceuticals and Dialysis Clinic Inc. She also has consulting agreements with Tricida Inc. and Omeros. A.S.L., J.C. and L.A.I. have a patent pending for precise estimation of GFR using multiple biomarkers (filed 15 Aug 2014; patent number PCT/US2015/044567). Tufts Medical Center, John Hopkins University and Metabolon Inc. have a collaboration agreement to develop a product to estimate GFR from a panel of markers. H.T. declares no competing interests. 